\title{
Prevalencia de caries según indice CEOD en escolares de 6 años Cuenca- Ecuador
}

\author{
Prevalence of caries according to ceod index in 6 years old school children of Cuenca- \\ Ecuador \\ Veléz Eleonor ${ }^{1 a b}$, Encalada Liliana ${ }^{1 a c}, F$ ernández María José ${ }^{1 a}$, Salinas Grecia ${ }^{1 a}$
}

\begin{abstract}
Resumen
Objetivo:Determinar el ceod en escolares de 6 años en la parroquia El Sagrario-Cuenca en el año 2016. Materiales y Métodos: Se realizó un estudio de tipo descriptivo, transversal, retrospectivo; la muestra estuvo constituida de 163 fichas epidemiológicas correspondientes a pacientes de 6 años, de los cuales el 55\% correspondió al sexo femenino y $45 \%$ de sexo masculino, pertenecientes a escuelas fiscales de la parroquia EI Sagrario. Resultados: Los resultados obtenidos mostraron que el índice ceod poblacional en los escolares de 6 años de la parroquia El Sagrario fue de 4,7, el cual no tuvo diferencias significativas entre el sexo masculino y femenino presentando valores de entre 4,5 y 4,9 respectivamente. Conclusiones: Basándose en los niveles de severidad de laOrganización Mundial de la Salud (OMS), la población de estudio presenta un alto riesgo de caries.
\end{abstract}

Palabras clave: Caries dental, índice, epidemiología. (Fuente: DeCS BIREME)

\begin{abstract}
Objective: Determine the ceod on school children of six years of age in the parish of El Sagrario-Cuenca in the year 2016. Materials and Methods: A descriptive, transversal, retrospectivestudy was carried out, the sample consisted of 163 epidemiological records corresponding to 6 -year-old patients, of which $55 \%$ were female and $45 \%$ were male, pertaining to public schools at El Sagrariodistrict. Results: The results obtained show that the average ceod index in the school children of six years of age at El Sagrario district is of 4.9. Which did not have significant differences between males and females presenting values between 4.5 and 4.9 respectively. Conclusions: Based on the levels of severity of World Health Organization(OMS), the study group shows a high risk of cavities.
\end{abstract}

Keywords:dental caries, Index, Epidemiology. (Source: MeSH NLM)

Recibido: 03 de noviembre de 2018

${ }^{1}$ Universidad Católica de Cuenca. Ecuador

a Odontólogo, b Especialista en Odontopediatria

c Especialista en Docencia Universitaria, Magister en Estomatología

Correspondencia:

María José Fernández Siguencia

Correo electrónico: siguenciafernandez.majo@hotmail.com
Aprobado: 25 de enero de 2019

Publicado: 31 de enero de 2019
Este es un artículo Open Access distribuido bajo la licencia Creative Commons Atribución-NoComercialCompartirlgual 4.0

Citar como: Veléz E, Encalada L, Fernandez MJ, Salinas G. Prevalencia de caries según indice CEOD en escolares de 6 años Cuenca- Ecuador. KIRU. 2019; 16(1): 27 - 31. https://doi.org/10.24265/kiru.2019.v16n1.04 


\section{INTRODUCCIÓN}

La caries dental no es una patología infecciosa clásica, al contrario, es una enfermedad compleja y multifactorial, producto de un desequilibrio en la biopelícula adquirida produciendo destrucción de tejidos calcificados del diente ${ }^{(1,2)}$.

Su etiología multifactorial incluye factores causales y factores de riesgo predisponentes. Dentro de los causales se describe: huésped susceptible, dieta alta en hidratos de carbono, y bacterias cariogénicas de la placa bacteriana, interactuando en un periodo de tiempo. Los factores de riesgo predisponentes que destacan son; conductuales, ambientales y genéticos ${ }^{(2,3)}$.

La caries constituye un problema generalizado a nivel de la población escolar, que presenta un alto grado de morbilidad y consecuente pérdida dental, de acuerdo con la información publicada por la OMS, se estima que aproximadamente del $60 \%$ al $90 \%$ de los escolares tienen esta patología a nivel mundial, por lo que Ecuador no es la excepción, y las cifras de prevalencia oscilan entre el 60 al $80 \%$ siendo los niños de edades tempranas los más afectados. Según datos del Ministerio de Salud Pública (MPS); existe un promedio de 4,62 en el ceoda los 6 años de edad ${ }^{(4,5)}$.

Existen varios indicadores epidemiológicos que nos permiten cuantificar y comparar la prevalencia de la enfermedad; el índice más usado a escala mundial en dientes temporales, debido a su fácil medición es el índice ceod. Éste índice valora los dientes primarios cariados, obturados y con indicación de extracción. Se aplica principalmente al grupo de edad entre los 5 a 6 años ${ }^{(3,6)}$.

El estudio se realizó con la finalidad de conocer la prevalencia de caries dental en los escolares de 6 años.

\section{MATERIALES Y MÉTODOS}

El presente es un estudio transversal, observacional descriptivo(7,8). La población de estudio fue de 163 fichas epidemiológicas de la parroquia EI Sagrario, que corresponden al total de fichas registradas en el estudio epidemiológico de salud bucal para esta parroquia. Los datos de éstas fichas fueron registrados una base de datos en el programa EPI-INFO versión 7.2. Se incluyeron las fichas epidemiológicas con su respectivo consentimiento y asentimiento informado. Se excluyeron aquellasque tenían incoherencias en los datos registrados.
El estudio buscó describir cuantitativamente el problema de caries dental en escolares de 6 años de edad, para esto se utilizó el índice ceod.

Para el diagnóstico de caries se utilizaron los criterios de diagnóstico recomendados por la Organización Mundial de Salud (OMS) ${ }^{(9)}$. Los datos registrados en las fichas fueron tomados por estudiantes previamente calibrados del último año de la carrera de odontología, cuyos valores obtenidos de la calibarcion fueron capa $>0.8$, para el registro se comenxo el examen bucal por el primer molar superior derecho (diente 1.6), continuando con el segundo molar superior derecho temporal (5.5), luego el número 5.4 y así sucesivamente hasta llegar al primer molar superior izquierdo (2.6), con el cual finalizó la examinación de las piezas dentales superiores. Luego continuó con las inferiores, con la misma secuencia que los superiores, posterior a esto verificaron la coherencia de los datos ${ }^{(10)}$.

El observador debió seguir las siguientes recomendaciones ${ }^{(11)}$ :

a) Evitar tocar la boca del paciente con los dedos. Inicialmente, cada diente se examina en forma visual para observación de áreas descalcificadas, opacidades de los bordes marginales y esmalte socavado en fosas y fisuras.

b) En caso de duda, usar la sonda periodontalrestringiendo su uso en caries avanzadas para evitar que el paciente sienta dolor innecesario.

c) Preguntar al paciente la razón de la extracción del diente, pero si la respuesta no permite obtener una conclusión el examinador seguirá su criterio clínico.

d) Dictar el código claramente, para evitar, errores de anotación.

e) Se deben examinar todas las superficies del diente.

Después de terminado el examen, los datos se consignaron en las casillas correspondientes, anotando el código respectivo que representa el Hallazgo clínico o del tipo de tratamiento requerido, según los respectivos códigos que se detallan en la tabla 1. 
Tabla 1. Codificación de la condición de los dientes OMS ${ }^{(10)}$.

\begin{tabular}{cl}
\hline Código & Condición del diente \\
\hline 0 & Sano \\
1 & Cariado \\
2 & Obturado con caries \\
3 & Obturado sin caries \\
4 & Perdido por caries \\
5 & Perdido por otro motivo \\
6 & Sellante o fisura obturada \\
7 & Pilar de puente, corona \\
& implante \\
8 & Diente permanente sin \\
& erupcionar \\
9 & No registrable \\
\hline
\end{tabular}

Para el cálculo del ceod; los códigos 1 y 2; se contabilizaron como el componente c "cariadas", el código 3 como el componente o "obturadas" y el código 4 como el componente e "extracciones indicadas" por caries únicamente(12).

Para consignar el código 1 el diente debe estar cavitado, en caso de que un diente esta obturado y cariado se consigna el diagnóstico más grave y en caso de que exista una restauración por medio de una corona se considera diente obturado(12).

Excluye los dientes extraídos con anterioridad, dientes con indicación de extracción por otra causa que no sea caries y la presencia de selladores ${ }^{(6)}$.

Para determinar el índice ceod se suma la frecuencia de códigos $1 ; 2 ; 3$ y 4 en cada paciente ${ }^{(12)}$.

Finalmente, para hallar el valor de ceod poblacional y por cada sexo, se calculó el promedio de los valores de todos los individuos, y se comparó los resultados con la tabla de valores de niveles de severidad de caries dental recomendada por la $\mathrm{OMS}^{(12) \text {. }}$

Tabla 2. Niveles de caries según $\mathrm{OMS}^{10}$

\begin{tabular}{ll}
\hline Valores & Niveles \\
\hline $0.0-1.1$. & Muy bajo \\
$1.2-2.6$ & Bajo \\
$2.7-4.4$ & Medio \\
$4.5-6.5$ & Alto \\
Mayor a 6.6 & Muy alto \\
\hline
\end{tabular}

Fuente: Petersen P. France:World Health Organization $2013^{(10)}$

\section{RESULTADOS}

Se evaluaron 163 escolares de 6 años,de los cuales, el $55 \%$ correspondió al sexo femenino y $45 \%$ al sexo masculino.

La prevalencia de enfermos fue de un $67,31 \%$ y los sanos fueron un $32,69 \%$.Tabla 3

Tabla 3. Distribución de la muestra en la parroquia El Sagrario en niños de 6 años.

\begin{tabular}{|c|c|c|}
\hline $\begin{array}{lll}\begin{array}{l}\text { Distribución } \\
\text { muestra }\end{array} & \text { de la } \\
\end{array}$ & $\mathbf{N}$ & $\%$ \\
\hline Sanos & 36 & 22 \\
\hline Enfermos & 127 & 78 \\
\hline Total & 163 & 100 \\
\hline
\end{tabular}

Con respecto al sexo fue más significativa en el sexo femenino con un $57 \%$ respecto al sexo masculino con un $43 \%$. Tabla 4

Tabla 4. Prevalencia de sanos y enfermos de acuerdo al sexo en escolares de 6 años de la Parroquia El Sagrario

\begin{tabular}{lcccc}
\hline \multirow{2}{*}{ Sexo } & \multicolumn{2}{c}{ Sanos } & \multicolumn{2}{c}{ Enfermos } \\
& N & $\%$ & N & $\%$ \\
\hline Masculino & 19 & 53 & 54 & 43 \\
Femenino & 17 & 47 & 73 & 57 \\
Total & 36 & 100 & 127 & 100 \\
\hline${ }^{*}$ Fuente propia & & & &
\end{tabular}

El índice ceod poblacional fue de 4.7; el cual no tuvo diferencias significativas entre el sexo masculino y femenino presentando valores de entre 4,5 y 4,9 respectivamente. Tabla 5

Tabla 5.Promedio de índice de ceod en la parroquia El Sagrario de acuerdo al sexo.

\begin{tabular}{lc}
\hline Sexo & Promedio ceod \\
\hline Femenino & 4,9 \\
Masculino & 4,5 \\
Comunitario & 4,7 \\
\hline *Fuente propia. &
\end{tabular}

En los niveles de severidad del ceod, se encontró la presencia de una severidad "alta" con un porcentaje del $28 \%$ del total de la población. Además, se observa que la frecuencia es uniforme en ambos sexos. Figura1. 


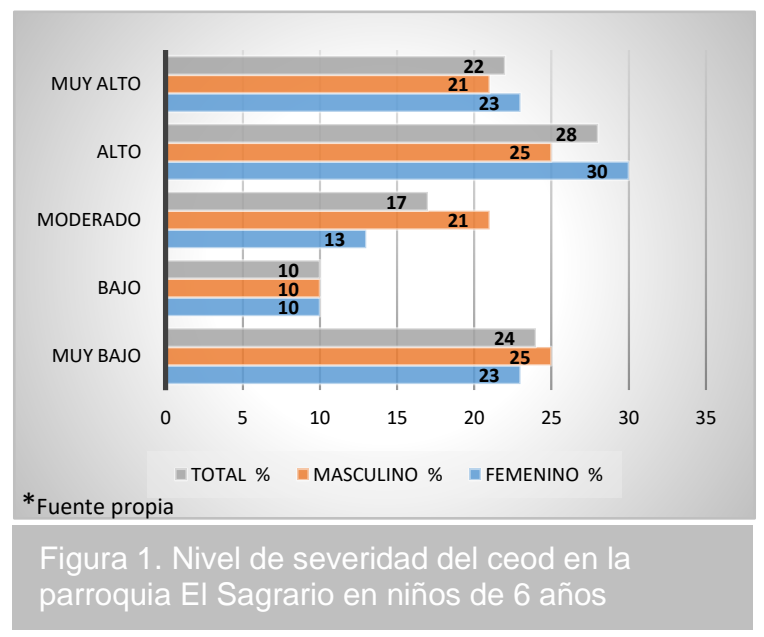

Finalmente en cuanto a la distribución de los componentes del ceod, los dientes cariados representan el $80 \%$; los extraídos el 6\%; y los obturados el 14\%. Observándose mayores porcentajes en el sexo femenino. Figura 2

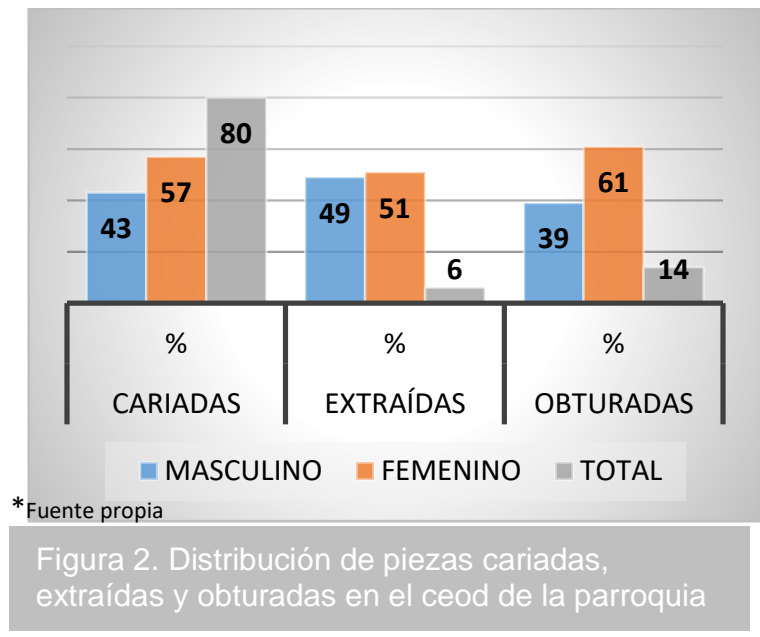

\section{DISCUSIÓN}

El ceod valora los dientes temporales cariados, obturados, y extracciones indicadas por caries. Para obtener el ceod en la parroquia EI Sagrario se examinaron 163 escolares de 6 años en 6 diferentes Unidades Educativas. Los datos obtenidos y analizados en éste estudio evidencian que el índice ceod comunitario encontrado fue de 4.71. Con respecto al sexo no se encontró diferencias significativas, entre el sexo masculino y femenino presentando valores de entre 4.52 y 4.86 respectivamente. Similar a los resultados encontrados en el estudio realizado por Villalobos y Medina ${ }^{(13)}$ en México,en el cual indicaron que, en la dentición temporal, el ceod fue 4,68; en las mujeres se registró un índice de 4.61 y en los hombres 4.75. En otro estudio realizado por
Villalobos y cols ${ }^{(14)}$ obtuvieron un índice ceod de 5.0; en el sexo femenino el índice correspondía al 4.93 y en el sexo masculino 5.12.

Por otro lado, se encontró una gran diferencia con otros estudios, como el realizado por Martínez y cols $^{(5)}$, en México, en el que obtuvieron un índice ceod de 1.88; para el sexo femenino de 1.90 y 1.87 para el masculino. Igualmente, en el estudio realizado por Zavarce(15) en Venezuela,se registró un promedio ceod de 1.96; el sexo masculino con 2.04 mientras que en el femenino fue de 1.54. Estos dos estudios reflejan un nivel de severidad de bajo riesgo, discrepando con nuestro estudio.

En cuanto a la prevalencia de caries dental en el presente estudio se evidenció el $78 \%$ de escolares enfermos. Valores similares reveló Cereceda y cols(16), en su investigación realizada en Chile, se encontró una prevalencia de 79,5\%. Así mismo en el estudio realizado por Medina(17) en México, se halló una prevalencia de $72,6 \%$. Sin embargo, al compararlo con Beltrán(18) existe una gran diferencia, ya que, en su estudio realizado en México, encontró una prevalencia en dentición primaria de $53.1 \%$.

Todos estos estudios reportan prevalencias de caries superiores al 50\%, lo que demestra que falta mucho por hacer en prevención de caries dental.

Con respecto a la prevalencia de caries según el género en éste estudio fue de $43 \%$ en el sexo masculino y $57 \%$ en el femenino, observándose una mayor prevalencia en el sexo femenino. Hallazgo similar al encontrado en el estudio realizado por Díaz y González ${ }^{(19)}$ en Colombia, registró la mayor prevalencia en las niñas con 56\%. Vásquez y Barrientos ${ }^{20}$ en su artículo mencionan que el sexo femenino tiene una mayor susceptibilidad para desarrollar caries dental, esto podría explicarse por la temprana erupción de los dientes en las mujeres, sin embargo, dicha teoría aún no tiene fundamento válido. La distribución del la muestra según sexo delpresente estudio explicaría esta diferencia en la prevalencia de caries, puesto queexistio un $10 \%$ mas para el sexo femenino en relación almasculino.

Con respecto a los resultados del índice ceod hallados en esta investigación se determinó que existe 613 dientes cariados (80\%), 47 dientes extraídos (6\%), y 107 dientes obturados (14\%). Comparados con el estudio realizado en México por Medina y cols(21) se observa que existe una similitud de ceod, ya que el $60.67 \%$ corresponde a dientes con caries, el $3.37 \%$ dientes indicados para extraccióny $7.19 \%$ dientes obturados, además que 
la mayor prevalencia fue de dientes cariados, seguidos de diente obturados y finalizando con dientes extraídos.

Esto demuestra que la caries ocupa el primer lugar en relación a enfermedades bucodentales, es importante mencionar también que la edad de la muestra estudiada esta en relación a una etapa de la vida donde el consumo de alimentos cariogenicos es alto y las medidas de higiene bucal son bajas, debido a la falta de conciencia de los niños en el autocuidado.

Se presentó ciertas limitaciones en el acceso a los centros educativos, debido a los permisos que se debieron obtener para la realización del estudio, sin embargo, estos se resolvieron de manera eficaz.

Es importante que las medidas preventivas sean aplicadas a los niños y a sus progenitores o tutores, a fin de disminuir esta patología y mejorar la calidad de vida de los niños.

Se concluye que la prevalencia de caries en la poblacion estudiada es alta, sin diferencia entre sexos. Basándose en los niveles de severidad establecidos por la OMS, el índice ceod poblacional hallado fue alto lo que aumenta el riesgo de caries.

\section{Contribuciones de autoría:}

MJFS y GS: Participaron en el diseño del estudio, recolección de datos y análisis de resultados. EVL y LEV, redactaron el artículo. Todos los autores aprobaron la versión final del artículo.

\section{Fuente de financiamiento: Autofinanciado}

Conflictos de interés: Los autores declaran no tener conflictos de interés en esta publicación.

\section{REFERENCIAS BIBLIOGRAFICAS}

1. Catalá M, Cortés $\mathrm{O}$. La caries una enfermedad que se puede prevenir. AnPediatrContin. 2014; 12(3).

2. Rojas S, Echeverria S. Caries temprana de infancia: ¿Enfermedad infecciosa? Revista Médica Clínica Las Condes. 2014 mayo; 25(3): 13-19.

3. Achahui $P$, Albinagorta M, Arauzo C, Cadenillas A, Céspedes Gary. Caries de infancia temprana: diagnóstico e identificación de factores de riesgo. Odontol. pediatr. julio-diciembre 2014; 13(2):119137.

4. Ministerio de Salud Pública. Protocolos odontológicos. Programa Nacional de Genética y Dirección. 2013.

5. Martínez K, Monjarás A, Patiño N, Loyola JP. Estudio epidemiológico sobre caries dental y necesidades de tratamiento en escolares de 6 a 12 años de edad de San Luis Potosí. Revista de Investigación Clínica. mayo-junio 2010; 62(3): 206213.
6. Piovano S, Squassl A, Bordoni N. Estado del arte de indicadores para la medición de caries dental. Revista de la Facultad de Odontología (UBA). 2010; 25(58): 26-30.

7. Villavicencio E. El tamaño muestral en tesis de post grado. ¿Cuantas personas debo encuestar? researchgate. Enero-Abril 2017; 2(1): 1-4.

8. Villavicencio $E$, et al. Pasos para la planificación de una investigación clínica. Oactiva. 2016;1(1):1-5.

9. Petersen P. Oral Health Surveys Basic Methods. World Health Organization. 2013; 5: 29-34

10. Ulate J, Rodríguez A y cols. Prevalencia de caries dental en niños y niñas escolares de 12 años de edad en Costa Rica, 2006. Rev. CientíficaOdont. 2011. 7(2).

11. Villavicencio E. Proyecto: Prevalencia de Caries en escolares de 12 años en Cuenca 2016.Researchgate

12. Reinoso N, Villavicencio E. Caries dental en escolares de 12 años de Sayausí, Cuenca, Ecuador.Revista OACTIVA, Enero 2016, (1).

13. Villalobos J, Medina C. Caries dental en escolares de 6 a 12 años de edad en Navolato, Sinaloa, México: experiencia, prevalencia, gravedad y necesidades de tratamiento. Biomédica, junio 2006, 26(2):224-233

14. Villalobos J, Medina C, Maupomé G, Pontigo A, Verdugo L. Caries dental en escolares de una comunidad del noroeste de México con dentición mixta y su asociación con algunas variables clínicas, socioeconómicas y sociodemográficas, Medigraphic, 2007, 59(04):256-267.

15. Zavarce E, Izzeddin R. Prevalencia de caries dental en menores de 5 años de edad. Estudio en Ciudad Hospitalaria Enrique Tejera, Valencia Estado Carabobo, Venezuela. Acta Odontológica Venezolana. 2014; 52(2).

16. Cereceda M, Faleiros S, Ormeño A, Pinto M, Tapia R, Diaz C, García H. Prevalencia de Caries en Alumnos de Educación Básica y su Asociación con el Estado Nutricional. RevChilPediatr. 2010; 81(1):28-36.

17. Medina Solís C, Maupomé G, Herrera M. Prevalencia de caries dental en escolares de 6-12 años de edad de León, Nicaragua. GacSanit. JulioAgosto 2005; 19(4)

18. Beltrán R, Cocom H, Casanova J, Vallejos A, Medina C, Maupomé G. Prevalencia de caries y algunos factores asociados en escolares de 6-9 años de edad en Campeche, México. RevBiomed. 2006; 17(1).

19. Díaz S, Gónzalez F. Prevalencia de caries dental y factores familiares en niños escolares de Cartagena de Indias, Colombia. Revista de salud pública. 2010 octubre; 12 (5).

20. Vásquez E, Barrientos M, Vásquez F. Prevalencia de caries dental en adolescentes: Asociación con género, escolaridad materna y estatus socioeconómico familiar. Revista CES Odontología. 2011; 24(1).

21. Medina S, Silva G, Palomera O, Hernández M.Prevalencia de caries dental del preescolar de la región costa norte de. Prevalencia de caries dental del preescolar de la región costa norte de Jalisco, México año 2010. Revista Tamé 2015.; 4(10). 